\title{
Respecter les normes et posséder un sens du spectacle en communication orale en sciences: les deux aspects du sentiment d'efficacité personnelle d'étudiants postsecondaires en sciences
}

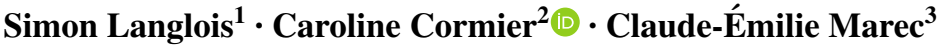

Accepted: 31 August 2021 /Published online: 4 November 2021

(C) Ontario Institute for Studies in Education (OISE) 2021

\begin{abstract}
Proficiency in oral communication is an important skill to develop in science. Central to its development is self-efficacy. In this study we examine two aspects of self-efficacy: first, standards and content, and second, showmanship. We looked at these aspects among students enrolled in a natural sciences program at the Quebec college level (a two-year post-secondary program in which students are usually between the ages of 17 and 19) in relation to their experiences of oral communication on scientific subjects. A sample of students responded to a questionnaire developed as part of this study on perceptions and attitudes towards communicating orally in the sciences (PACOS), first at the start of their program $(n=1292)$ and then at the end $(n=179)$. A subsample of this group $(n=26)$ also participated in an interview for deeper analysis. The results, coupled with those from a previous study, show that the two aforementioned aspects of self-efficacy are distinct but complementary. Moreover, we observed that they are linked to other subcomponents of one's attitude towards scientific oral communication-namely anxiety, pleasure, perception of relevance, and context. Finally, we noted that the standards and content aspect of self-efficacy remained consistent over time, while the sense of showmanship increased among participants between the start and end of their studies.
\end{abstract}

Résumé L'habileté à communiquer à l'oral est une compétence importante à développer en science. Central au développement de celle-ci, le sentiment d'efficacité personnelle (SEP) est étudié dans cette recherche en accord avec les deux aspects qui le composent, soit le SEP normes et contenus et le SEP sens du spectacle. Ces deux aspects du SEP ont été étudiés chez des étudiants du programme

Caroline Cormier

caroline.cormier@claurendeau.qc.ca

Simon Langlois

simon.langlois@collegemv.qc.ca

1 Cégep Marie-Victorin, 7000, rue Marie-Victorin, Montréal, Québec) H1G 2J6, Canada

2 Cégep André-Laurendeau, 1111, rue Lapierre, Montréal (arr. Lasalle) (Québec H8N 2J4, Canada

3 Faculté Des Sciences de L'éducation, Université du Québec À Montréal, Pavillon Paul-Gérin-Lajoie, 1205, rue Saint-Denis, Montréal, Québec) H2X 3R9, Canada 
collégial québécois de Sciences de la nature (programme post-secondaire de 2 ans, entre 17 et 19 ans habituellement), en lien avec leurs expériences en communication orale sur des sujets scientifiques. Un échantillon d'étudiants de ce programme a répondu au questionnaire PACOS (Perceptions et attitude en communication orale en sciences) développé dans le cadre de cette recherche, d'abord au début de leurs études collégiales $(n=1292)$, puis à la fin $(n=179)$. Un sous-échantillon d'entre eux $(n=26)$ a de plus participé à un entretien d'approfondissement. Les résultats, couplés à ceux d'une étude antérieure, montrent que les deux aspects du SEP sont bien distincts, mais complémentaires. En outre, nous avons observé que les deux aspects du SEP sont reliés aux autres sous-composantes de l'attitude envers la communication orale scientifique, nommément l'anxiété, le plaisir, la perception de la pertinence et la dépendance au contexte. Finalement, nous avons noté que le SEP normes et contenu semblait stable dans le temps, tandis que le SEP sens du spectacle avait augmenté entre le début et la fin des études collégiales chez nos participants.

Mots-clés Communication orale $\cdot$ Communication scientifique $\cdot$ Sentiment d'efficacité personnelle . Attitude $\cdot$ Enseignement des sciences

\section{Problématique}

De nombreux auteurs soulignent l'ubiquité de la communication orale dans la vie quotidienne comme dans tous les secteurs de la vie professionnelle (Blanchet, 2017; McLaren, 2019; Mercer-Mapstone \& Matthews, 2017). Ils énoncent clairement que s'exercer à la communication orale au niveau postsecondaire devient un enjeu éducatif et social. Bien que la communication orale soit prescrite au programme du collégial et préconisée dans toutes les disciplines (MEES, 2021) sous forme d'interactions variées, elle semble demeurer marginale ou «trop souvent oubliée ou négligée» (Dumais, 2017, p. 13), généralement par manque d'outils pédagogiques destinés aux enseignants. Blanchet (2016) et Dumais (Dumais, 2017; Dumais \& Ostiguy, 2019) appellent à considérer la communication orale comme une compétence à développer pour que les étudiants puissent la mettre à profit dans leurs études et plus tard dans leur domaine professionnel.

Dans le champ disciplinaire des sciences en particulier, d'autres voix suggèrent aussi que la communication orale en sciences (COS) soit enseignée aux étudiants dès les niveaux préuniversitaires (Chan, 2011; Edmondston et al., 2010). Ces recommandations rejoignent les préoccupations des autorités éducatives au Canada, comme en Australie, aux États-Unis ou en Angleterre (Kulgemeyer \& Schecker, 2013; MercerMapstone \& Kuchel, 2015). Alors que les disciplines comme la biologie, la chimie ou les mathématiques mettent généralement l'accent sur l'appropriation de la connaissance technique (Bjorklund \& Colbeck, 2001; Brownell et al., 2013; Cleveland \& Reinsvold, 2017), l'étude de Kulgemeyer (2018) démontre que la maitrise du contenu notionnel (content knowledge) ne suffit pas à réaliser une communication orale en science de qualité. Dans leur étude sur la perception de la communication orale en science d'étudiants en biotechnologie, Edmondston et al. (2010) rapportent que ces étudiants obtiennent leur diplôme sans vraiment comprendre ce que représente la COS, sa fonction ni sa pertinence. Brownell et al. (2013) abondent dans le même sens et constatent que même les scientifiques seraient peu formés pour transmettre des concepts scientifiques à un auditoire non initié. Pour McLaren (2019), les habiletés à communiquer oralement jouent non seulement un rôle important dans la réussite scolaire des étudiants, mais elles contribuent aussi à accroitre leur confiance en eux autant que leur capacité à structurer et présenter des arguments convaincants.

Parmi les mécanismes intellectuels et affectifs qui concourent à la production d'une communication orale, le sentiment d'efficacité personnelle tient une fonction primordiale (Jones \& Leagon, 2014), car il module autant le flux des émotions que les processus cognitif et motivationnel qui 
conduisent à l'action (Boyer, 2012). Dès lors, le SEP apparait comme un concept incontournable pour mieux comprendre comment les étudiants appréhendent la COS. Or, le SEP en situation de COS ne semble pas constituer un objet fréquent de recherche (Dumais, 2017; Kulgemeyer \& Schecker, 2013). En effet, dans notre recension de littérature scientifique, nous n'avons pas rencontré d'études portant particulièrement sur le SEP d'élèves ou d'étudiants en situation de COS. Si certaines études analysent le lien entre le SEP et la communication orale (Boyer et al., 2018; Demir, 2017; Dwyer \& Fus, 2002), d'autres traitent de la compétence en COS en lien avec le contenu notionnel (Kulgemeyer $\&$ Schecker, 2013), des conceptions de la COS (Edmondston et al., 2010) ou encore d'attitude et de motivation envers la COS (McLaren, 2019). Il y a donc une absence d'étude sur la relation entre SEP et COS, alors que celle-ci nous paraît essentielle pour identifier et saisir les éléments manifestes et sous-jacents qui participent à la réalisation d'une COS.

Par ailleurs, au-delà du contenu proprement scientifique, le fait de devoir prendre la parole dans une langue différente de la langue dans laquelle on s'exprime à la maison pourrait être une variable importante à considérer lors de l'étude de la réalisation d'une COS. En effet, les locuteurs dont le français est la langue seconde peuvent avoir plus d'anxiété à s'exprimer en français étant donné la perception négative qu'ils ont de la qualité de leur vocabulaire ou de leur prononciation (Papin, 2019). Dans une étude portant sur des étudiants en ingénierie dont la langue maternelle n'était pas la même que la langue d'enseignement, Khatib et Maarof (2015) rapportent que les étudiants démontrent un SEP à l'oral dans la langue d'enseignement de plus en plus élevé au fil des années passées à étudier dans cette langue seconde. Dans la même étude, il est de plus noté que le SEP à l'oral des étudiantes est supérieur à celui des étudiants, ce qu'on explique par l'intérêt plus grand démontré par les femmes envers les langues, ce qui est cohérent avec d'autres observations sur la différence de SEP à l'oral en langue seconde selon le genre (Mercer \& Williams, 2014). Toutefois, le SEP mesuré par Kahtib et Maarof porte sur l'habileté à parler - avec ses pairs, avec ses professeurs - et non pas à réaliser une communication orale formelle sur un sujet scientifique. En bref, il semble bien qu' aucune étude portant précisément sur l'effet de la langue parlée à la maison (lorsque différente de la langue d'enseignement) ou du genre sur le SEP en COS n'ait été répertoriée dans les écrits de recherche.

Sur ce terrain de recherche, une étude antérieure menée par Langlois et Cormier (soumis) auprès d'étudiants du niveau collégial (deux ans d'étude de 17 à 19 ans généralement) a interrogé leurs perceptions et leur attitude envers la COS par le biais du questionnaire Perceptions et Attitude des étudiants envers la communication orale en sciences (PACOS). Ce questionnaire conçu par les auteurs comprend entre autres des items sur le SEP en situation de communication orale en sciences. Une analyse factorielle a conduit les auteurs à des résultats inédits dans ce domaine de recherche: ceux-ci ont révélé un SEP divisé en deux aspects que les auteurs ont nommés normes et contenu et sens du spectacle. Les auteurs définissent le SEP normes et contenu par le sentiment de réussir une COS en s'exprimant clairement, en mesurant sa voix, sa diction, en observant les normes discursives propres à la communication en sciences et en présentant de façon juste et précise le contenu scientifique qui sous-tend la communication, alors que le SEP sens du spectacle réfère au sentiment de susciter l'intérêt de son auditoire en étant dynamique, conscient de sa communication gestuelle et par la capacité du locuteur d'adapter son discours au public visé. Ces deux aspects du SEP apparaissent tous deux essentiels pour développer la compétence en COS, alors que la «base» de la communication en science repose sur le contenu et que l'adaptation du discours en fonction de l'auditoire est nécessaire pour bien se faire comprendre (Kulgemeyer, 2018).

En identifiant un SEP divisé en deux aspects, nos résultats nous ont amenés à concevoir un modèle théorique des Perceptions et de l'attitude envers la COS, désigné par PACOS, qui intègre ces deux aspects. Envisagés comme des facteurs de stress, de risque ou de succès en situation de COS, les deux aspects du SEP apportent un nouveau regard dans l'étude de la COS et soulignent la pertinence de les examiner plus en profondeur. 
C'est dans le prolongement de cette première étude du SEP caractérisé par ses deux aspects que se situe la présente étude. Elle vise à mieux comprendre comment s'exprime ce SEP, notamment à préciser l'influence d'autres caractéristiques propres aux étudiants sur chacun des volets du SEP en COS, et à vérifier jusqu'à quel point ces deux volets du SEP des étudiants changent au fil des études collégiales. Étudier et documenter la manière dont les étudiants préuniversitaires du programme québécois de Sciences de la nature de niveau collégial se perçoivent et se sentent en situation de COS pourrait certainement ouvrir des pistes de réflexion quant à la formation à leur offrir et affirmerait la pertinence d'un tel enseignement.

\section{Cadre conceptuel}

En nous inspirant du modèle de l'attitude envers les sciences de van Aalderen-Smeets et al. (2012, 2013), nous avons appuyé notre modèle PACOS sur les trois dimensions de l'attitude: croyances cognitives, états affectifs et perception de contrôle dans laquelle figure le SEP inscrit sous ses deux aspects: SEPnc et SEPss.

Notre modèle PACOS, à la Fig. 1, est ainsi constitué de trois dimensions fortement liées entre elles. Les croyances cognitives réfèrent à la perception de la pertinence accordée à la COS dans le quotidien, dans les études ou dans un projet professionnel, qui se conjugue à la représentation que l'étudiant se fait de la COS et à l'utilité qu'il lui confère. Les états affectifs s'expriment sous la forme du plaisir ou de l'anxiété éprouvés envers la réalisation d'une COS.

Dans notre modèle et à l'instar du modèle de l'attitude de van Aalderen et de ses collaboratrices (2012), le SEP se loge dans la dimension des «perceptions de contrôle» (Perceived control). Pour ces auteures, le SEP «résulte de la combinaison de sentiments et de croyances au sujet de facteurs internes (capacité, connaissances et expérience envers les sciences) qui influence le sentiment d'être en contrôle en ce qui concerne l'exécution d'actions particulières» (Van Aalderen-Smeets et al., 2012, p. 168, traduction libre).

Concept clé de la théorie sociocognitive de Bandura (2007), le SEP se définit comme la perception ou le jugement d'un individu sur sa capacité à réaliser une tâche et à produire les résultats envisagés. Il fait partie d'un système de croyances qui agissent sur les décisions du sujet et l'évaluation des actes

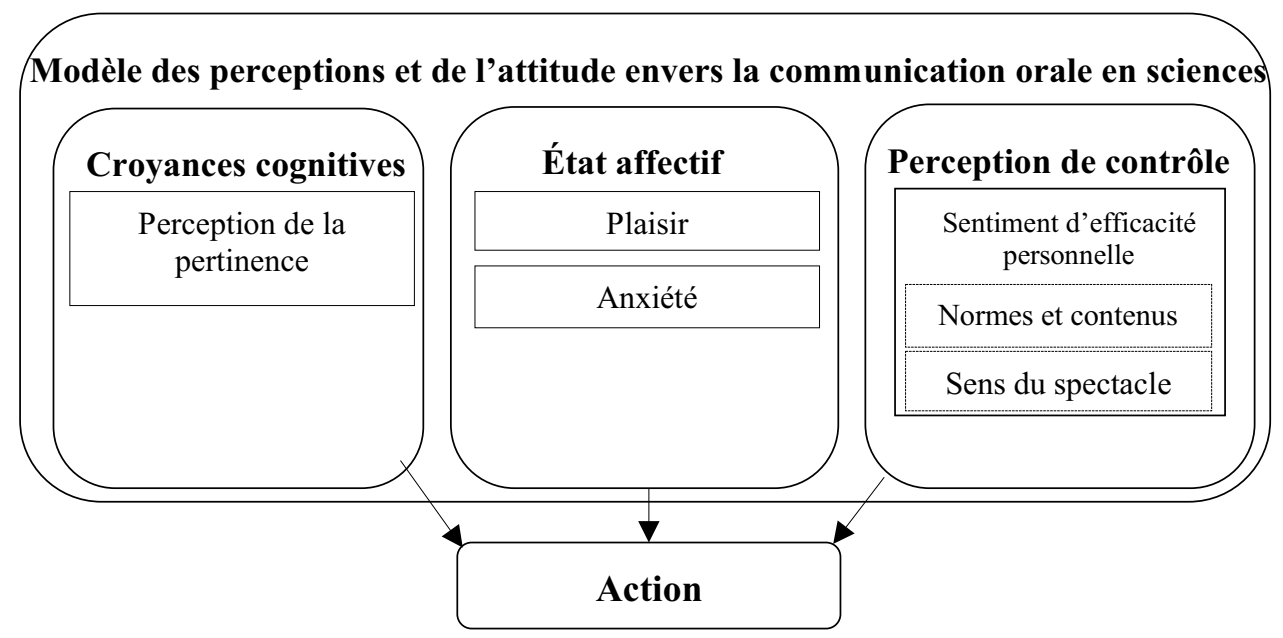

Fig. 1 Modèle théorique des Perceptions et de l'attitude envers la communication orale en sciences, adapté du modèle théorique de van Aalderen-Smeets et al. (2012) 
posés, sur la persévérance ou la démission, l'engagement dans des activités ou l'abandon, etc. (Gaudreau et al., 2012). Le SEP est considéré comme «un des déterminants majeurs du comportement» (Boyer, 2012, p. 3).

Dans le cas qui nous intéresse, la perception de contrôle opère sur la réalisation d'une COS entre autres par le «contrôle» qu'elle exerce sur les actions à poser et par son impact sur les éléments de stress et de difficulté qu'induit la tâche. Nous souscrivons à cette approche conceptuelle, à la nature du SEP considéré comme une perception de contrôle dans la mesure où toute activité ou situation de COS implique autant les sentiments et les croyances sur soi que les fonctions intellectuelles.

De ce modèle, c'est le SEP sous ses deux aspects, normes et contenuetsens du spectacle, qui sert de fondement à notre recherche. Il est examiné en tant que sous-composante de l'attitude et étudié en lien avec les autres sous-composantes des dimensions cognitive et affective.

Le SEP est d'autant plus prédictif de la performance d'une tâche qu'il s'inscrit dans un domaine d'activités spécifiques (Bandura, 2009), en l'occurrence la COS dans laquelle l'étudiant fonde son action à partir de ses capacités intellectuelles et leur complémentarité affective. Le SEP, dans toute sa complexité, est le mécanisme qui participe de façon importante au fonctionnement du sujet (Bandura, 2009).

Le SEP, sous ses deux aspects normes et contenu et sens du spectacle émerge comme un des éléments clés qui détermine la qualité de la communication orale en sciences. Il se trouve lié aux sentiments positifs (plaisir, intérêt, etc.) ou négatifs (crainte, anxiété, déplaisir, etc.) et aux croyances personnelles quant à ses capacités, ses connaissances et ses expériences.

La distinction entre les deux SEP nous apparait porteuse de sens en contexte de COS: un étudiant qui possède un SEP normes et contenu (SEPnc) élevé se considère capable de fournir un discours scientifique dont les propos sont justes, en utilisant la terminologie appropriée et dans une langue correcte. Un étudiant qui possède un fort SEP sens du spectacle (SEPss) perçoit quant à lui qu'il peut rendre sa présentation intéressante, en employant des stratégies non verbales et en adaptant son propos à son auditoire, notamment. Les deux aspects du SEP peuvent ainsi résonner de façon bien distincte dans la perception de soi d'un individu, même s'il demeure qu'ils sont fortement corrélés (Langlois \& Cormier, soumis).

Au-delà de la présence du SEP dans la dimension de contrôle, il est à noter la participation commune de toutes les sous-composantes de l'attitude vers l'action (dimension comportementale), elle-même située en tant que dimension externe aux composantes de l'attitude de notre modèle PACOS. Selon la théorie de l'action planifiée (theory of planned behavior) de Ajzen et Fishbein (2005) auquel le modèle renvoie, le comportement identifié à l'action est à envisager comme le résultat ou la conséquence (outcome) de l'attitude (Van Aalderen-Smeets et al., 2012). Dans le cadre d'une COS, cette action représente autant les comportements adoptés par l'étudiant pendant l'exposé que les actes qui préparent à celui-ci.

\section{Relations entre sep et autres construits (ou sous-composantes) de l'attitude}

L'anxiété est souvent associée à une appréhension de prendre la parole devant un auditoire. Dwyer et Fus (2002) ont indiqué une corrélation entre l'appréhension (communication apprehension), le sentiment d'efficacité personnelle et la perception de sa compétence à parler en public (self-perceived public speaking competence). Une forte appréhension entrainerait un SEP faible, mais qui peut être corrigé par une formation appropriée. Au même titre que l'anxiété, le plaisir influencerait le SEP et se voit associé à l'intérêt et à la curiosité pour les sciences (Alsop \& Bencze, 2014). Plaisir et intérêt combinés à la réussite scolaire entrainent en général un engagement positif et durable dans les activités scientifiques (Osborne et al., 2003), en l'occurrence dans des activités de COS.

Enfin, des études montrent que le SEP de l'étudiant est inhérent à l'apprentissage et à la réussite scolaire (Masson \& Fenouillet, 2013) et se révèle un facteur déterminant pour la réussite d'une activité spécifique (Bandura, 2007), comme une COS. Par la place prépondérante que le SEP occupe en situation 
de COS, il demeure le facteur principal à étudier. Cependant, ce sont également les relations qu'il entretient avec les sous-composantes de l'attitude autour desquelles il s'articule qu'il est pertinent d'analyser.

\section{Objectifs}

Les objectifs de la présente étude visent à mieux comprendre la complexité des deux aspects du SEP en COS. Notre premier objectif consiste à mesurer la corrélation entre les deux aspects du SEP et entre chacun de ces aspects et les autres sous-composantes du modèle du PACOS (plaisir, anxiété, et perception de la pertinence), puis à évaluer le lien entre le SEP et les caractéristiques sociodémographiques de notre échantillon. Notre deuxième objectif vise à décrire les facteurs évoqués par les étudiants lors d'un entretien téléphonique pour expliquer ce qui influence selon eux leur SEPnc et leur SEPss en COS. Notre troisième objectif est de rendre compte de l'évolution des deux aspects du SEP en COS en comparant ces scores au début et à la fin des études collégiales.

\section{Méthodologie}

Pour répondre pleinement à nos trois objectifs, nous avons opté pour une méthodologie mixte en vue d'une meilleure compréhension de notre objet d'étude (Fortin \& Gagnon, 2015). Nous avons utilisé un devis séquentiel explicatif (Creswell \& Creswell, 2018) par lequel l'analyse des données quantitatives sert à répondre partiellement aux objectifs et permet d'orienter la prise de données qualitatives, puis nous avons procédé à l'intégration des données quantitatives et qualitatives au moment de l'interprétation des résultats.

\section{Procédures d'échantillonnage et de collecte des données}

La population à l'étude était composée d'étudiants du programme collégial québécois de Sciences de la nature, un programme préuniversitaire de deux ans (quatre sessions) comprenant des cours de biologie, de chimie, de physique et de mathématiques, pour un total de 12 cours de formation spécifique en plus de 15 cours de formation générale (dont français et anglais langue seconde). Dans le programme de Sciences de la nature, le cours de français porteur de la compétence 4EFP, «produire différents types de discours oraux et écrits liés au champ d'études de l'élève» (MEES, 2017a, p. 15), inclut des éléments portant sur la communication orale. Toutefois, comme nous l'avons indiqué plus tôt, les pratiques enseignantes dans ce cours sont disparates entre les cégeps et entre les enseignants d'un même cégep (Blanchet, 2017).

En 2015-2016 (année la plus récente dont les statistiques étaient disponibles), la population des étudiants du programme de Sciences de la nature comptait 25898 étudiants (MEES, 2021). De cette population, un échantillon de 1292 étudiants volontaires provenant de sept cégeps publics francophones de la grande région de Montréal a été recruté par l'entremise d'enseignants-ressources dans chacun des cégeps participants. Les étudiants volontaires étaient inscrits en première session du programme de Sciences de la nature.

La procédure de collecte de données s'est effectuée en trois temps sur une période de deux ans. La Fig. 2 illustre les trois temps de la collecte de données, le type d'instruments utilisés et la taille de l'échantillon. 


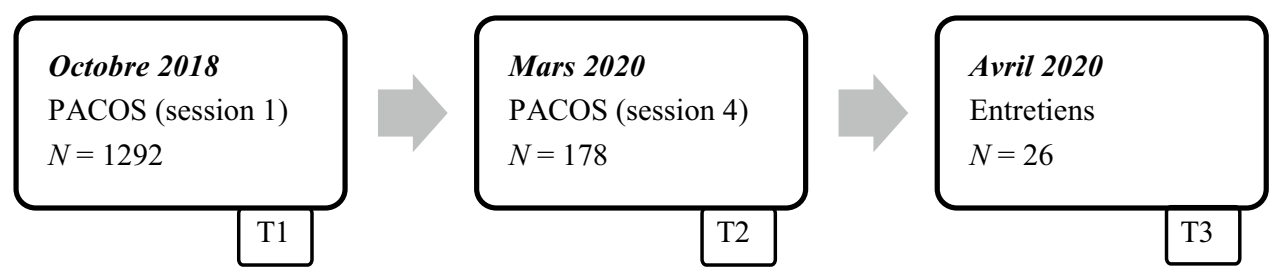

Fig. 2 Résumé de la collecte de données en trois temps

Au temps 1 (T1): en première session d'études collégiales, les 1292 étudiants volontaires ont rempli le questionnaire PACOS en format papier, en classe. Ces données permettent de répondre à l'objectif 1 qui porte sur les corrélations entre les deux aspects du SEP et entre chacun de ces aspects et les autres sous-composantes du modèle du PACOS, puis à évaluer le lien entre le SEP et les caractéristiques sociodémographiques de notre échantillon.

Au temps 2 (T2): en dernière session d'études collégiales, 179 étudiants qui faisaient partie de l'échantillon de départ ont rempli à nouveau le questionnaire PACOS, mais soumis en grande majorité sous forme électronique, compte tenu de la situation sanitaire imposée par la COVID-19. Le taux de réponse pour le temps $\mathrm{T} 2$ a été de $14 \%$. Ces données, combinés à ceux du temps T1, permettent de répondre à l'objectif 3 qui porte sur l'évolution des deux aspects du SEP.

Précisons qu'entre ces deux passations de questionnaire, il n'y a pas eu d'intervention pédagogique particulière en référence à la COS, sinon les activités orales prévues habituellement par les professeurs selon les cours.

Au temps 3 (T3): Afin de répondre à l'objectif 2 (qui vise à décrire les facteurs évoqués par les étudiants pour expliquer ce qui influence selon eux leur SEPnc et leur SEPss en COS), un sous-échantillon de 26 étudiants, parmi ceux ayant répondu au questionnaire au temps T2 seulement ( 9 étudiants) ou aux temps T1 et T2 (17 étudiants), a participé aux entretiens individuels, conduits par téléphone, par l'un des trois chercheurs. Un échantillonnage stratifié avec répartition proportionnelle a été réalisé (Blair \& Blair, 2014), les strates étant issues d'une analyse par grappes des scores de SEP mesurés au temps T2 $(n=179)$. L'analyse par grappes respecte les critères établis par Olsen et Lie (2011), dont celui de retirer aléatoirement $30 \%$ des données pour vérifier la stabilité des profils obtenus. Trois profils ont été dégagés de cette analyse (silhouette 0,5). La composition des profils et la distribution des étudiants interviewés en fonction de leur appartenance à chacun des profils sont décrites dans le Tableau 1.

Comme l'indique ce tableau, la stratification des étudiants choisis pour être interviewés donne une distribution très semblable à celle des étudiants ayant répondu au questionnaire PACOS à la session d'hiver 2020 $\left(\chi^{2}[2]=0,708, p=0,701\right)$. Ainsi, en référence à la nature de leur SEP, les étudiants interviewés sont représentatifs de ceux de l'ensemble des étudiants ayant répondu au questionnaire; leurs impressions et réponses à nos questions sont donc probablement représentatives de celles de l'ensemble de l'échantillon.

Tableau 1 Comparaison de la composition de l'échantillon stratifié des étudiants interviewés par rapport à l'ensemble de l'échantillon ayant répondu au questionnaire au temps T2

\begin{tabular}{llll}
\hline Profil & SEP & $\begin{array}{l}\text { Nombre dans l'échantillon } \\
(\%)\end{array}$ & $\begin{array}{l}\text { Nombre } \\
\text { interviewés } \\
(\%)\end{array}$ \\
\hline 1 & Élevé & $42(23,5 \%)$ & $4(15,4 \%)$ \\
2 & Moyen & $90(50,3 \%)$ & $15(57,7 \%)$ \\
3 & Faible & $47(26,3 \%)$ & $7(26,9 \%)$ \\
Total & & $179(100 \%)$ & $26(100 \%)$ \\
\hline
\end{tabular}




\section{Instruments de mesure et méthodes d'analyse}

Deux outils de collecte de données ont été utilisés dans cette recherche: le questionnaire PACOS et le protocole d'entretien individuel semi-dirigé.

\section{Questionnaire PACOS}

Le questionnaire PACOS (Perceptions et attitudes envers la communication orale scientifique) a été développé pour cette recherche. Il s'agit d'un questionnaire comptant 34 items à échelle de Likert à 4 niveaux (1: totalement en désaccord, 2: en désaccord, 3: en accord et 4: totalement d'accord). Nous avons choisi de fixer le nombre de niveaux à quatre, car il a été montré que les jeunes étudiants peuvent avoir de la difficulté à bien s'évaluer sur une échelle à six niveaux ou plus (Smith et al., 2003); nous avons aussi préféré un nombre pair de niveaux, de façon à éliminer la valeur neutre qui peut agir comme valeur refuge (Smith et al., 2003).

Lors de l'élaboration du questionnaire PACOS, des analyses factorielles exploratoire avec méthode d'extraction en composantes principales (Field, 2017) (indice KMO de 0,964 et signification du test de Bartett $\chi^{2}(496)=2528, \mathrm{p}<0,001$ ) et confirmatoire (RMSEA $<0,05$; CFI $=0,94$; SRMR $=0,05$ et $\left.\chi^{2}(517)=1735,43, \mathrm{p}<0,001\right)$ ont permis de déterminer que les items du questionnaire se regroupent en cinq facteurs (Langlois \& Cormier, soumis) qu'on retrouve aussi dans le modèle PACOS présenté plus haut. Ce modèle explique $67,5 \%$ de la variance.

Dans la dimension des états affectifs, on trouve des items sur l'anxiété ( 5 items; $\alpha=0,95)$ et le plai$\operatorname{sir}(11$ items; $\alpha=0,91)$ en situation de COS. Dans la dimension des croyances cognitives, on trouve la perception de la pertinence associée à la COS ( 5 items; $\alpha=0,77)$. Dans la dimension de la perception de contrôle, on trouve les deux aspects du SEP: le SEP normes et contenu ( 8 items; $\alpha=0,84$ ), qui réfère au sentiment d'être un communicateur qui maitrise son contenu et s'exprime correctement, et le SEP sens $d u$ spectacle ( 5 items; $\alpha=0,87$ ), qui réfère au sentiment d'être un communicateur capable de susciter l'intérêt du public. Le temps de complétion moyen du questionnaire en ligne était de 6 min $45 \mathrm{~s}$ avec un écart-type de $2 \min 45 \mathrm{~s}$.

Des items sociodémographiques complétaient le questionnaire, dont l'âge, le genre (féminin, masculin, autre/préfère ne pas répondre) et la langue le plus souvent parlée à la maison (réponse ouverte).

\section{Entretiens}

L'entretien individuel de type semi-dirigé fondé sur des questions ouvertes avait pour but de laisser les étudiants s'exprimer au sujet de la COS pour pouvoir analyser le sens qu'ils donnent à leur pratique et à «mettre en évidence les systèmes de valeur et les repères normatifs à partir desquels ils s'orientent et se déterminent» (Blanchet et Gotman, 2014, cités par Boutin, 2018, p. 22). Un protocole d'entretien appuyé sur les objectifs de l'étude et les résultats obtenus au questionnaire PACOS a été établi par les chercheurs et testé auprès de 10 étudiants avant d'être soumis aux répondants. Les éléments de l'entretien s'orientaient sur le respect des normes de présentation propres aux sciences (normes linguistiques et discursives) relativement à l'aspect normes et contenu du SEP et sur la façon de susciter l'intérêt lors d'un exposé oral (normes communicatives) relativement à l'aspect sens du spectacle. Les entretiens se sont déroulés au téléphone et ont duré entre 20 et $25 \mathrm{~min}$. Leur transcription a été effectuée par les chercheurs eux-mêmes. 


\section{Méthode d'analyse des entretiens}

Les données textuelles issues des entretiens ont été soumises à une analyse de contenu. Dans un premier temps, les chercheurs ont codé séparément trois cas chacun (neuf au total) pour identifier des catégories émergentes pour chacun des thèmes (SEPnc et SEPss) et arriver à une grille d'analyse commune. À partir de cette grille, ils ont codé séparément les 17 autres entretiens restants (1101 unités de sens au total). Un processus de contre-codage a ensuite été réalisé (Fortin \& Gagnon, 2015; Karsenti \& Savoie-Zajc, 2018), qui a mené à un accord interjuges supérieur à $80 \%$. Pour s'assurer d'un consensus sur l'ensemble des unités de sens, toutes les divergences restantes ont été discutées entre les chercheurs, qui se sont entendus sur un codage définitif sans désaccord. Cet exercice a produit 14 catégories émergentes pour chaque SEP. En comparant les catégories selon le poids des énoncés qu' elles contiennent, nous avons pu établir un ordonnancement catégoriel (Karsenti \& Savoie-Zajc, 2018). Pour chaque thème SEPnc et SEPss, nous avons ainsi réduit le nombre de catégories à 4 en ne retenant que celles qui comptaient le plus d'occurrences mentionnées, selon un seuil minimal établi à $20 \%$ des étudiants dont les propos rejoignent la catégorie.

\section{Résultats}

De cette collecte de données, nous avons obtenu des données portant sur le SEP des étudiants en COS. Les résultats issus de ces données sont présentés dans les sections suivantes, en fonction de l'objectif auquel ils réfèrent.

Objectif 1: scores du SEP et relation avec les autres scores mesurés par le questionnaire PACOS et les variables sociodémographiques recueillies.

Le questionnaire PACOS mesure deux aspects distincts du SEP: le SEP normes et contenu (SEPnc) et le SEP sens du spectacle (SEPss). Rappelons que le SEPnc, comme nous le définissons dans le contexte de la COS, est l'aspect du SEP qui porte sur les normes linguistiques (qualité de la langue parlée) et le contenu notionnel, tandis que le SEPss porte davantage sur l'intérêt suscité chez l'auditoire et par l'adéquation du discours à cet auditoire. Nous avons comparé les scores de ces deux aspects du SEP entre eux et avons trouvé qu'ils sont fortement corrélés l'un à l'autre $(r=0,649, \mathrm{p}<0,01)$. Le score moyen au SEPnc, dont la moyenne est 2,90 (écart-type $=0,45$ ), est significativement plus élevé que celui au SEPss, en moyenne de 2,64 (écart-type $=0,64$ ), avec un effet de grande taille (Cohen, 1988) (t [1291] = 17,37; $\left.\mathrm{p}<0,001, \eta^{2}=0,20\right)$. Ceci indique que les étudiants se perçoivent en moyenne davantage comme des communicateurs qui maitrisent bien le contenu et les normes de présentation plutôt que des communicateurs foncièrement intéressants.

Nous avons ensuite étudié quelles variables étaient corrélées à ces deux aspects du SEP. Ces analyses ont été menées sur les données recueillies chez les étudiants de première session collégiale $(n=1292)$ Tableau 2.

Tableau 2 Indices de corrélation de Pearson (r) entre les scores aux facteurs de SEP et les autres scores mesurés par le questionnaire PACOS

\begin{tabular}{|c|c|c|c|}
\hline \multirow[t]{2}{*}{ Facteurs du SEP } & \multicolumn{3}{|c|}{ Autres facteurs du questionnaire PACOS } \\
\hline & Anxiété & Plaisir & $\begin{array}{l}\text { Perception de } \\
\text { la pertinence }\end{array}$ \\
\hline $\mathrm{SEP} \ll$ normes et contenu» & $-0,285^{* *}$ & $0,352^{* *}$ & $0,368^{* *}$ \\
\hline $\mathrm{SEP}$ «sens du spectacle» & $-0,501^{* *}$ & $0,531^{* *}$ & $0,351^{* *}$ \\
\hline
\end{tabular}


Tableau 3 Comparaison des scores aux deux aspects du SEP selon le genre des étudiants (tests $t$ )

\begin{tabular}{|c|c|c|c|c|c|}
\hline \multirow[b]{2}{*}{ Variable } & \multicolumn{2}{|c|}{$\begin{array}{l}\text { Filles } \\
(n=737)\end{array}$} & \multicolumn{2}{|c|}{$\begin{array}{c}\text { Garçons } \\
(n=523)\end{array}$} & \multirow[b]{2}{*}{$p$} \\
\hline & $\bar{M}$ & ÉT & $\bar{M}$ & ÉT & \\
\hline SEPnc & 2,92 & 0,48 & 2,97 & 0,48 & 0,056 \\
\hline SEPss & 2,65 & 0,65 & 2,70 & 0,63 & 0,132 \\
\hline
\end{tabular}

Tous les indices de corrélation entre les facteurs du questionnaire PACOS et les deux volets du SEP sont significatifs à $\mathrm{p}<0,001$. Cependant, on peut constater que l'indice de corrélation est plus élevé entre le SEP «sens du spectacle» et les facteurs de la dimension de l'état affectif (anxiété: $r=-0,501$ et plaisir: $r=0,531$ ) qu'entre le SEP «normes et contenu» et les facteurs de la dimension de l'état affectif (anxiété: $r=-0,285$ et plaisir: $r=0,352$ ), semblant indiquer une composante plus affective au SEP «sens du spectacle», ce qui nous paraît cohérent avec la définition que nous faisons de cet aspect du SEP à l'oral.

Pour poursuivre la distinction entre le SEPnc et le SEPss en COS, certains aspects de la variabilité sociodémographique des étudiants ayant participé à la recherche ont fait l'objet d'analyses: d'abord, le genre des étudiants et ensuite, la langue qu'ils parlent le plus souvent à la maison.

Tout d'abord, le Tableau 3 qui suit montre que le SEPnc et le SEPss s'exprimaient de la même façon pour les garçons et pour les filles, la différence n'étant pas significative pour ces deux aspects du SEP ( $p>0,05$ dans les deux cas).

On voit dans ce tableau que le SEP des garçons est marginalement plus élevé que celui des filles, mais ce de façon non significative.

Ensuite, et en continuité avec le fait que la communication orale demeure un objet d'apprentissage de la discipline du français, nous avons cherché à vérifier si la langue parlée à la maison avait une influence sur le SEP en COS. Rappelons d'abord que la langue d'enseignement de tous les participants à cette recherche était le français. Lorsqu'on leur a demandé quelle langue ils parlaient à la maison (question ouverte), nous avons pu répertorier, outre le français $(68,8 \%$ des répondants) plus de trente autres langues, les plus fréquentes étant l'arabe (12,7\%), l'anglais (4,2\%), l'espagnol (3,2\%), le vietnamien (1,2\%) et le roumain $(1,1 \%)$. Selon que la langue le plus souvent parlée à la maison était ou non le français, le SEP des étudiants s'exprimait différemment, comme on le voit au tableau suivant Tableau 4.

Tableau 4 Comparaison des scores aux deux aspects du SEP selon la langue le plus souvent parlée à la maison (français ou autre langue) (tests $t$ )

\begin{tabular}{llllllll}
\hline & \multicolumn{2}{l}{$\begin{array}{l}\text { Français } \\
(\mathrm{n}=920)\end{array}$} & & & \multicolumn{2}{l}{$\begin{array}{l}\text { Autre langue } \\
(\mathrm{n}=416)\end{array}$} \\
\cline { 2 - 3 } \cline { 6 - 8 } Variable & $\mathrm{M}$ & ÉT & & $\mathrm{M}$ & ÉT & $\mathrm{t}$ & $\mathrm{p}$ \\
\hline SEPnc & 2,96 & 0,47 & & 2,86 & 0,50 & 3,679 & $<0,001$ \\
SEPss & 2,67 & 0,65 & & 2,67 & 0,65 & $-0,136$ & 0,892 \\
\hline
\end{tabular}


Il est à noter que seul le SEPnc diffère entre les étudiants qui parlent le français à la maison et ceux qui parlent une autre langue: en effet, le SEPnc des premiers est significativement plus élevé que celui des seconds (avec un effet de taille moyenne, $\eta^{2}=0,104$ ). Le SEPss est quant à lui identique, peu importe la langue parlée à la maison. L'absence de distinction entre les garçons et les filles et la différence significative au SEPnc selon la langue parlée à la maison seront discutées plus avant dans la section discussion.

Présentés ci-dessous, les résultats des entretiens associés au deuxième objectif tentent d'expliquer plus en profondeur les éléments explicatifs du SEPnc et du SEPss, et ce, du point de vue de l'étudiant. Il est à noter que comme les résultats de l'objectif 1 n'indiquent aucune différence significative entre le SEPnc et le SEPss selon le genre, aucune analyse différenciée n'a été réalisée selon cette variable pour l'objectif 2. Par contre, la langue émerge quant à elle comme une catégorie explicative du SEPnc.

\section{Objectif 2: description des éléments explicatifs du sepnc et du sepss}

Lors du processus d'analyse, nous avons procédé à une hiérarchisation des catégories émergentes selon leur ordre d'importance pour ensuite en réduire le nombre à quatre (Dionne, 2018). Les quatre catégories émergentes pour chaque aspect du SEP sont listées et détaillées.

\section{Catégories émergentes explicatives du sepnc (normes et contenu)}

Notre question d'entretien concernait la perception qu'a l'étudiant de la qualité de ses explications lors d'un exposé oral. Notre analyse de contenu a dégagé les catégories émergentes suivantes: contenu discursif, langue, préparation et SEPss, présentées dans le Tableau 5 et illustrées par un exemple de réponse.

Contenu discursif: Cette catégorie comprend deux aspects: la connaissance du contenu et la complexité du sujet. Pour 19 étudiants, maitriser le sujet abordé et les termes scientifiques propres à ce sujet est essentiel à une bonne communication. Comprendre le sujet à présenter est une condition pour être capable de bien l'expliquer pour 13 étudiants: «dès que je comprends la matière, c'est très facile pour moi de l'expliquer» (2062). Pourtant, trois étudiants signalent que comprendre ne signifie pas forcément

Tableau 5 Catégories émergentes du SEPnc avec des extraits de réponses d'étudiants à la question «Que peux-tu dire à propos de la qualité de tes explications lors d'un exposé oral ?»

\begin{tabular}{|c|c|c|c|}
\hline $\begin{array}{l}\text { Catégories émer- } \\
\text { gentes }\end{array}$ & $\begin{array}{l}\text { Nombre } \\
\text { d'étudiants }\end{array}$ & $\begin{array}{l}\text { Nombre } \\
\text { d'énoncés }\end{array}$ & Exemples de réponses d'étudiants \\
\hline Contenu discursif & 20 & 40 & $\begin{array}{l}\text { - La plupart du temps, je maitrise mon contenu ça me permet de bien trans- } \\
\text { mettre la matière (2060) } \\
\text { - Il faut que tu aies une meilleure connaissance de ton sujet en sciences par } \\
\text { rapport à français }(3697)\end{array}$ \\
\hline Langue & 23 & 37 & $\begin{array}{l}\text { - J'essaie de parler fort et clairement pour que les gens comprennent le plus } \\
\text { possible (3684) } \\
\text { - Le choix de mots, je dois encore améliorer ça beaucoup plus. Les termes } \\
\text { scientifiques, surtout en sciences ( } 3038)\end{array}$ \\
\hline Préparation & 17 & 33 & $\begin{array}{l}\text { - Je révise, je regarde toute la matière, je demande à des amis si ça a du } \\
\text { sens ce que je dis et après je me pratique, je le refais trois fois ( } 3518) \\
\text { - J'essaie de me préparer le mieux possible, d'expliquer chaque point, que } \\
\text { ça coule entre chaque idée ( } 3684)\end{array}$ \\
\hline $\begin{array}{l}\text { Adaptation du } \\
\text { contenu selon } \\
\text { l'auditoire } \\
\text { (SEPss) }\end{array}$ & 8 & 12 & $\begin{array}{l}\text { - Quand je connais mon sujet, je vais donner tous les détails pour faire } \\
\text { comprendre un concept, sans aller dans les détails inutiles ( } 3684) \\
\text { - Je pense que mes explications sont correctes, j'essaie de les simplifier } \\
\text { (3670) }\end{array}$ \\
\hline
\end{tabular}


savoir expliquer: «je vais bien comprendre, mais expliquer c'est moins évident» (2305). Neuf étudiants évoquent plus spécifiquement que la complexité inhérente au contenu rend les présentations plus difficiles en sciences que dans d'autres sujets ou d'autres cours.

Langue: La langue est mentionnée par une vaste majorité d'étudiants (23 sur 26) en réponse à la question sur ce qui fait que leurs explications sont de qualité. Le choix et la qualité du vocabulaire et l'utilisation de termes scientifiques justes sont des moyens de livrer de bonnes explications. Quatorze étudiants évoquent leurs qualités vocales pour affirmer que leurs explications sont de bonne qualité, ceci étant souvent associé, dans un même énoncé, à la façon dont ils captent et maintiennent l'intérêt. Cependant, des blocages apparaissent pour ceux dont le français n'est pas la langue maternelle, ce qui rend la verbalisation plus ardue ou la prononciation plus difficile, ce qui entraine parfois un débit trop rapide généré par le stress. Ces propos rappellent les résultats rapportés plus haut d'un SEPnc plus faible chez les étudiants dont le français n'était pas la langue parlée à la maison.

Préparation: La qualité des explications dépend aussi de la préparation de l'exposé. La pratique avant la présentation est une stratégie pour la plupart des étudiants. Les uns répètent devant autrui pour vérifier la cohérence de leur discours et des informations, apprennent par cœur ou répètent pour chronométrer le temps de parole et couper le surplus de matière. D'autres s'assurent de maitriser le contenu, de se préparer parce qu'on veut «être sûr de ne pas faire d'erreurs, de ne pas dire des faussetés» (2355).

Adaptation du contenu selon l'auditoire (SEPss): Six étudiants ont recours à des stratégies de vulgarisation pour donner de bonnes explications. Le souci de se faire comprendre demeure présent. Bien connaître son sujet permet d'improviser au besoin, tout en évitant le stress.

Anxiété, plaisir, perception de la pertinence: Les étudiants ne relient pas ou très peu leur SEPnc à l'anxiété ( 2 fois), au plaisir ( 0 fois), la perception de la pertinence ( 0 fois) ou aux résultats académiques (5 fois).

\section{Catégories émergentes explicatives du sepss (sens du spectacle)}

Notre question d'entretien concernait la manière dont l'étudiant suscite l'intérêt de son auditoire. Notre analyse de contenu a fourni quatre catégories émergentes relatives aux normes et contenu, aux techniques pour capter l'attention, aux techniques de vulgarisation et à la dépendance au contexte. Ces catégories émergentes sont présentées dans le Tableau 6 accompagnées d'un exemple de réponse.

Tableau 6 Catégories émergentes du SEPss avec des extraits de réponses d'étudiants à la question «Comment fais-tu pour susciter l'intérêt?»

\begin{tabular}{|c|c|c|c|}
\hline $\begin{array}{l}\text { Catégories émer- } \\
\text { gentes }\end{array}$ & $\begin{array}{l}\text { Nombre } \\
\text { d'étudiants }\end{array}$ & $\begin{array}{l}\text { Nombre } \\
\text { d'énoncés }\end{array}$ & Exemples de réponses d'étudiants \\
\hline $\begin{array}{l}\text { Normes et contenu } \\
\text { (SEPnc) }\end{array}$ & 23 & 38 & $\begin{array}{l}\text { - Je recueille les informations, je cherche les informations intéres- } \\
\text { santes, pertinentes et qui sont nouvelles (2871) } \\
\text { - J'essaie de parler assez fort, d'avoir des intonations (3685) }\end{array}$ \\
\hline $\begin{array}{l}\text { Techniques pour } \\
\text { capter l'attention }\end{array}$ & 22 & 51 & $\begin{array}{l}\text { - Je maintiens le contact visuel avec mon auditoire, j'essaie de faire un } \\
\text { balayage visuel. Je regarde tout le monde et un peu plus souvent le } \\
\text { professeur (3681) }\end{array}$ \\
\hline $\begin{array}{l}\text { Techniques de } \\
\text { vulgarisation }\end{array}$ & 17 & 43 & $\begin{array}{l}\text { - Je simplifie beaucoup les termes }[\ldots] \text {, je cherche des synonymes ou } \\
\text { des définitions (2638) }\end{array}$ \\
\hline $\begin{array}{l}\text { Dépendance au } \\
\text { contexte }\end{array}$ & 17 & 24 & $\begin{array}{l}\text { - Ça dépend vraiment de la matière qu'on présente [...] un discours sur } \\
\text { un sujet inspirant et tout, c'est plus facile susciter l'intérêt (3038) } \\
\text { - Je ne regarde pas beaucoup mon PowerPoint (3670) }\end{array}$ \\
\hline
\end{tabular}


Normes et contenu (SEPnc): Une vaste majorité d'étudiants (23 sur 26) estime susciter l'intérêt de l'auditoire, ce qui relève du SEPss, en évoquant un élément appartenant plutôt aux normes et contenu, donc rattaché théoriquement au SEPnc. Il s'agit plus spécifiquement pour 16 étudiants de susciter l'intérêt par leurs qualités vocales (voix forte ou sans monotonie) propres à la prosodie. De plus, 7 étudiants parmi les répondants misent sur le contenu notionnel, sur la qualité de l'information et sur la maitrise du contenu pour rendre l'exposé intéressant. Pour eux, ceci passe par une bonne préparation de l'information et du contenu à présenter.

Techniques pour capter l'attention: La principale technique évoquée par les étudiants pour capter l'attention de l'auditoire est d'utiliser le langage non verbal, ce qui inclut la posture, la gestuelle et le contact visuel. Ce dernier est d'ailleurs spécifiquement mentionné par 12 étudiants pour qui «balayer la classe» ou «regarder dans les yeux» permet de créer ce lien avec l'auditoire; à l'opposé, deux étudiants avouent avoir de la difficulté à maintenir le contact visuel: «quand je suis stressée [...] mon espace visuel ou mes yeux vont être plus concentrés sur l'écran que sur les personnes qui me regardent» (2871). En ce qui concerne le support visuel, d'ailleurs, 10 étudiants disent que c'est grâce à lui qu'ils parviennent à capter l'attention: avec une présentation PowerPoint de qualité, ces étudiants croient que le public sera plus attentif.

Techniques de vulgarisation: Elles font référence à des stratégies verbales pour rendre le propos plus clair, plus accessible, pour faire «comprendre» (10 étudiants). Pour 13 étudiants, c'est souvent une explication «dans leurs mots», des termes simplifiés ou des définitions quand le concept est peu connu ou un exemple qui illustre le propos: «quand j'utilise des mots plus précis que mon auditoire ne va pas comprendre, j'essaie d'expliquer par des images, des graphiques» (2062). Pour quatre répondants, vulgariser en faisant référence à des notions étudiées sert aussi à retenir l'attention: «je faisais beaucoup appel à des notions [déjà] vues pour que les personnes soient interpelées» (3698).

Dépendance au contexte: Cette catégorie regroupe des facteurs externes à la qualité des explications ou à la manière personnelle de susciter l'intérêt de l'auditoire. Ces facteurs externes sont envisagés par l'étudiant comme étant hors de son contrôle, mais exerçant une influence sur la réussite de sa COS. Parmi ces facteurs externes, le sujet même de la présentation a un effet sur l'intérêt que portera l'auditoire: 15 étudiants y sont sensibles. Ainsi, susciter l'intérêt de l'auditoire dépend d'un «sujet inspirant pour l'auditoire» (3038), de sa capacité à comprendre les concepts abordés ou d'une discipline qui lui est familière. Quant au recours à un support matériel (petits cartons, diaporama, etc.), sept étudiants affirment ne pas en dépendre pour livrer leur exposé oral. Ils connaissent bien leur texte et disent posséder une capacité à improviser, à développer une idée sans se servir d'un quelconque support matériel.

Toujours en référence à la dépendance au contexte, certains étudiants mentionnent des caractéristiques de l'auditoire comme un élément déterminant leur capacité à susciter l'intérêt, par exemple s'ils connaissent ou non leurs collègues de classe: «le fait que je connaisse moins ces personnes-là pourrait un peu influencer la façon dont je parle ou dont j'attire l'attention, alors qu'en général, je suis capable

Tableau 7 Scores moyens de SEPnc et de SEPss des répondants au questionnaire PACOS $(n=179)$ mesurés au début et à la fin de leur parcours collégial

\begin{tabular}{llllll}
\hline & \multicolumn{2}{l}{ Score au début du collégial } & & \multicolumn{2}{l}{$\begin{array}{l}\text { Score à la fin du } \\
\text { collégial }\end{array}$} \\
\cline { 2 - 3 } \cline { 5 - 6 } Variable & $\mathrm{M}$ & & M & ÉT \\
\hline SEPnc & 3,09 & 0,44 & & 3,15 & 0,45 \\
SEPss & 2,78 & 0,68 & & 2,94 & 0,62 \\
\hline
\end{tabular}


de susciter l'attention» (2060). D'autres étudiants témoignent que, malgré leurs efforts, l'auditoire ne leur semble pas intéressé en général.

Anxiété, plaisir, perception de la pertinence: Les étudiants ne relient pas ou très peu leur SEPss à l'anxiété (4 fois), au plaisir ( 0 fois), à la perception de la pertinence ( 0 fois), ou aux résultats académiques (0 fois).

\section{Objectif 3: évolution du sep en cos pendant les études collégiales}

Comme mentionné précédemment, le questionnaire PACOS a été complété au début (première session) et à la fin (quatrième session) du parcours collégial d'une cohorte d'étudiants de sciences. Nous avons voulu vérifier si les scores de SEP variaient, pour ces étudiants, au fil de leurs études collégiales. Le Tableau 7 présente les scores descriptifs des deux aspects du SEP, et ce, pour les deux temps de mesures. Étant donné que la valeur médiane se trouve à 2,5, un score moyen entre 1 et 2,5 indique une faible perception du SEPnc ou du SEPss, tandis qu' un score entre 2,5 et 4 indique une forte perception d'eux-mêmes quant à leur SEPnc ou SEPss.

Que ce soit au début ou à la fin de leur parcours collégial, les scores moyens indiquent un SEPnc et un SEPss élevés chez ces étudiants. De plus, malgré la petite augmentation observable entre les moyennes de SEPnc au début et à la fin du collégial, un test de Student pour échantillons appariés indique que cette différence n'est pas significative $(\mathrm{t}[178]=-1,86 ; \mathrm{p}=0,07)$. Par contre, le test de Student effectué sur le SEPss montre qu'il y a une différence significative entre le début et la fin des études collégiales chez les étudiants questionnés $(\mathrm{t}[178]=-4,20 ; \mathrm{p}<0,001 ; \mathrm{d}=0,24)$ avec une taille d'effet faible (Cohen, 1988). Ainsi, il apparaît que si le SEPnc est stable dans le temps, les étudiants témoignent d'une meilleure perception d'eux-mêmes en COS en termes de «sens du spectacle» après quatre sessions dans le programme de sciences de la nature.

\section{Discussion}

Dans notre étude, nous avons étudié le SEP en l'évaluant dans une activité spécifique, comme le préconise Bandura (2007), soit en COS et plus précisément lors d'un exposé oral. De ces résultats, nous tirons certains éclaircissements conceptuels des deux aspects du SEP.

\section{Différences et absences de différences en sep selon les variables sociodémographiques}

L'absence de différence entre les garçons et les filles en termes des deux aspects du SEP en COS (SEPnc et SEPss) nous apparaît intéressante à discuter. Dans les écrits de recherche, des études sur la différence entre les garçons et les filles en termes de SEP ont été réalisées dans des contextes éducatifs variés. Parmi celles-ci, certains écrits rapportent que le SEP des garçons est plus élevé que celui des filles, par exemple, face à la réussite d'examens de secondaire 4 (Dumont et al., 2003) ce que les auteurs expliquent par la plus grande inquiétude que les filles ressentent face aux examens. Au contraire, dans le contexte d'un travail collectif en français au primaire, Desombre et ses collaborateurs (2016) ont plutôt observé que le SEP des filles était plus élevé que celui des garçons. Ceci peut s'expliquer, selon ces auteurs, par la plus grande efficience en travail collectif des filles. Dans le contexte de notre étude, l'absence de corrélation entre le SEP et le genre des étudiants peut peut-être s'expliquer par la façon différente dont les filles et les garçons réussissent à développer un SEP élevé. Usher et Pajares (2006) suggèrent que les expériences de maîtrise, le facteur le plus important dans le développement du SEP, représentent le 
facteur déterminant du SEP chez les garçons, tandis que chez les filles, une combinaison des expériences de maîtrise (mastery experiences) et de la persuasion par autrui (social persuasion) fondent leur SEP. Leur postulat est que les filles sont plus sensibles à la rétroaction reçue (des pairs, des enseignants...), qui influence beaucoup leur perception de soi et par conséquent leur SEP.

L'absence de différence dans les SEP des garçons et des filles dans le contexte précis de notre recherche pourrait s'expliquer par la tâche sur laquelle nous avons mesuré ces SEP: la communication orale en sciences. Il semble en effet que dans les disciplines comme les sciences et les mathématiques, le SEP des garçons est plus élevé (Vrugt et al., 2009), tandis que dans les disciplines comme le français, le SEP des filles est plus élevé (Desombre et al., 2016; Skaalvik \& Skaalvik, 2004). Notre contexte se situant précisément à l'interface entre le français (au sens du français oral) et les sciences, c'est peut-être pourquoi le SEP des garçons ne se distingue pas de celui des filles, ni pour l'aspect SEPnc, ni pour l'aspect SEPss. Il demeure que la communication orale est une sous-discipline du français qui implique des réactions affectives souvent très différentes des autres activités pédagogiques réalisées en français, comme la rédaction de textes par exemple. L'absence de différence entre les garçons et les filles en termes de SEP en communication orale est peut-être due à cet aspect particulier du contexte que nous avons étudié, dont les résultats se rapprochent de ceux de Demir (2017) pour la communication orale.

En ce qui concerne la différence de SEPnc selon la langue parlée à la maison (français ou autre langue) et l'absence d'une telle différence pour le SEPss, ceci semble confirmer la nature distincte des deux aspects du SEP que nous proposons. Le SEPnc portant davantage sur la perception de s'exprimer correctement, il est cohérent que les étudiants dont la langue habituelle n'est pas la langue d'enseignement puissent percevoir leur habileté en la matière moins favorablement que ceux qui s'expriment au quotidien dans la même langue que celle dans laquelle ils font leurs exposés oraux à l'école. Dans une étude réalisée sur SEP à l'écrit d'étudiants universitaires américains, Huerta et ses collaboratrices (2017) ont rapporté que le SEP des locuteurs non natifs était significativement plus faible que celui dont l'anglais (langue de l'enseignement dans leur contexte) était la langue maternelle. Si les tâches d'écriture et de communication orale sont différentes, il demeure qu'on peut facilement les mettre en parallèle par rapport à la langue maternelle: les locuteurs non natifs ont un SEP plus faible lorsque vient le temps de communiquer, par écrit ou oralement, que les locuteurs natifs. Ce qui surprend, dans nos résultats, c'est plutôt l'absence de différence au niveau du SEPss selon la langue parlée à la maison. Ceci montre bien que les exigences de la COS sont différentes lorsqu'elles concernent la qualité de la langue, les habiletés discursives et le contenu, et lorsqu'elles concernent plutôt la capacité à susciter l'intérêt chez l'auditoire.

\section{Interprétation des résultats concernant le SEPnc}

Pour donner des explications de bonne qualité lors d'un exposé, les étudiants ont visiblement recours à la maitrise de leurs connaissances. Celles-ci s'acquièrent par des recherches approfondies, une préparation adéquate dans le choix des informations autant que dans le choix de la terminologie scientifique. Il semble que, selon les étudiants, l'exposé en sciences soit exigeant sur le plan des informations qui doivent être justes, qu'il doive rapporter des faits et se fonder sur des méthodologies précises. Aussi, le SEPnc relève d'une détermination de respecter les normes discursives propres aux sciences, de bien connaitre son sujet, pour être capable de bien l'expliquer. Cette préoccupation de bien expliquer pour se faire comprendre rejoint l'utilisation de techniques de vulgarisation pour susciter l'intérêt de l'auditoire (SEPss), montrant ainsi le caractère complémentaire des deux aspects du SEP.

Pour maitriser le contenu notionnel, les étudiants ont développé des stratégies, comme s'investir dans la recherche d'informations justes, s'assurer d'une terminologie appropriée, se préparer adéquatement, répéter leur texte. Ce comportement répond à la volonté de livrer son discours conformément à ce qui est attendu. L'étudiant dirige ses actions en vue de l'exposé, mobilise ses capacités intellectuelles et émotives. Par là, il 
montre sa capacité à agir sur l'activité d'exposé oral, malgré la complexité de la matière ou les difficultés que pourrait poser la langue. Ce faisant, il forge son sentiment d'être en contrôle de l'activité à venir.

\section{Interprétation des résultats concernant le SEPsS}

Le SEPss s'appuie sur le non verbal et sur des techniques de vulgarisation. Les techniques du non verbal semblent être conscientes et mesurées. Pourtant, si le contact visuel permet de maintenir le contact avec l'auditoire, nous pouvons imaginer cependant qu'il ne puisse pas garantir son intérêt ou la persistance de son intérêt. Pour leur part, les techniques de vulgarisation montrent un souci de se faire comprendre, de s'adapter à l'auditoire. Simplifier, expliquer et donner des exemples sont des recours «pédagogiques» pour assurer la compréhension de tous, mais aussi pour s'assurer de passer la matière. D'ailleurs, c'est souvent par le regard porté sur l'auditoire que les étudiants sentent le besoin de vulgariser, quand ils soupçonnent une incompréhension de sa part. Ce qui suggère, d'une part, une capacité du présentateur à décoder les messages de son auditoire et, d'autre part, une habileté à utiliser les mécanismes propres à l'interaction alors que la coopération communicative dans un contexte d'exposé oral est minime (Koch $\&$ Oesterreicher, 2001). L'exposé oral «impose au sujet parlant une verbalisation complexe et hautement intégrée» (Koch \& Oesterreicher, 2001, p. 591) ainsi qu' une norme prescriptive. Nous constatons ici que nos répondants savent utiliser des marqueurs linguistiques pour rendre leur discours accessible.

La langue et la qualité des informations contribuent aussi au SEPss pour rendre le contenu de l'exposé intéressant. Ainsi, ces facteurs participent d'une performance réussie en COS. De plus, concernant le support visuel (diaporama PowerPoint) en COS, nos répondants disent ne pas en dépendre et n'en faire qu'un usage essentiellement utilitaire. Ils expriment ainsi une perception de contrôle, laissant supposer que les textes sont maitrisés ou suffisamment bien appris pour être livrés sans aide. Bien qu'ils n'aient pas reçu de formation spécifique en COS, ils reconnaissent que le support visuel ne doit rester qu'un matériel d'appoint, alors qu'une étude menée par Applebee et ses collaborateurs (2018) auprès d'étudiants préuniversitaires en chimie indiquait leur difficulté à s'affranchir de leur support visuel (PowerPoint) en début de formation sur la COS. Cette même étude rapporte toutefois qu'en fin de formation, les étudiants avaient acquis une meilleure utilisation du support visuel.

\section{Relation entre les deux aspects du sep émergeant des entretiens et du modèle PACOS}

Les résultats obtenus montrent la complémentarité indéniable du SEPss et du SEPnc, ce qui suggère qu'ils seraient bel et bien des sous construits du SEP lors d'une COS. Selon les travaux de Bandura (2007), il n'y aurait qu'un seul SEP contextualisé à une tâche spécifique. Or, notre étude en précise la définition dans ce contexte particulier de la COS en le définissant par ses deux aspects.

Alors que l'indice de corrélation entre le SEPnc et le SEPss est très élevé, l'analyse des entretiens nuance ce résultat. Elle suggère que la relation entre les deux aspects du SEP est asymétrique. Ainsi, la maitrise de la langue et du contenu notionnel (SEPnc) sont plus souvent évoqués comme facteurs explicatifs de l'efficacité à communiquer un message (SEPss) que dans la direction opposée, soit que la capacité à bien communiquer des contenus à un auditoire (SEPss) permet aux étudiants de se percevoir comme efficaces dans la maitrise du contenu et de la langue.

Ceci pourrait s'expliquer par l'idée qu'ont certains étudiants que le sens du spectacle est quelque chose de plutôt inné ou naturel, tandis qu'un exposé oral en science qui se veut efficace et qui respecte les normes discursives peut être réalisé par toute personne s'étant bien préparée.

Il se peut aussi que le sens du spectacle s'applique moins dans le cadre d'un exposé en science qui demande rigueur et sérieux. Les énoncés des entretiens témoignent de cette perception qu'ont les étudiants qu'un exposé oral en science rapporte des faits, des analyses, des données chiffrées et non des opinions, comme le permettent des exposés en français, par exemple. L'exposé oral en science vise 
avant tout à diffuser un contenu notionnel qui peut utiliser des techniques de vulgarisation au bénéfice de l'auditoire. Cette perception semble s'accorder avec une représentation traditionnelle de la science: le contenu discursif est fondamental et le sens du spectacle lui serait subordonné.

Kulgemeyer (2018) arrive au même constat alors que des étudiants (fin du secondaire) inscrits à des cours de physique présentaient une bonne maitrise du contenu notionnel (content knowledge) mais possédaient toutefois de faibles aptitudes de communication. Kulgemeyer rappelle que la «maitrise du contenu notionnel est assurément un mauvais prédicteur de l'habileté à communiquer» (Kulgemeyer, 2018, p. 105, traduction libre) et que les étudiants devraient en être conscients. Comme cet auteur, nous convenons que les habiletés communicatives sont parties prenantes de l'exposé oral. Cependant, nous rappelons que les habiletés communicatives en référence au langage non verbal sont fortement présentes dans le discours de nos étudiants. Si ces derniers reconnaissent une large place au contenu discursif dans la réalisation d'une COS, ils ne négligent pas pour autant certains principes clés de la communication.

\section{Interprétation de l'évolution du sepnc et du sepss entre le temps T1 et le temps T2}

En ce qui concerne l'évolution du SEP en COS, les entretiens semblent indiquer que le score de SEPnc n'évolue pas durant les études collégiales. Les étudiants interprètent cette constance par un nombre insuffisant d'oraux réalisés au collégial. Ils ajoutent aussi que le contenu disciplinaire à maitriser augmente en complexité entre le début et la fin de leurs études collégiales, ce qui rend les COS plus difficiles à réaliser.

L'évolution du SEPss pourrait s'expliquer selon les étudiants par le fait que l'auditoire est connu des présentateurs à la fin de leur parcours collégial (T2), ce qui diminue le stress et permet de se «concentrer plus sur sa façon de présenter» (3672). D'autres mentionnent aussi la maturation naturelle des étudiants qui écoutent les exposés: «Les gens sont plus adultes. Ils t'écoutent, ils ne sont pas indifférents. Même s'ils ne sont pas intéressés, ils ne vont pas le montrer» (3684).

En comparant les habiletés de communications d'étudiants préuniversitaires de $2^{\mathrm{e}}$ et $3^{\mathrm{e}}$ années, Mercer-Mapstone et Kuchel (2015) soulignent que la confiance augmente significativement au fil des années. À tout le moins pour le SEPss, nous rejoignons ce constat. Il semble cependant que ce ne soit pas le cas pour le SEPnc.

\section{Vers un nouveau modèle des perceptions et de l'attitude envers la communication orale en sciences}

Comme l'indiquent les analyses statistiques, il existe bien des corrélations entre chacune des souscomposantes du modèle des perceptions et de l'attitude envers la COS. La perception de la pertinence, du plaisir et de l'anxiété, le SEP normes et contenu ainsi que SEP sens du spectacle exercent des influences complexes qui façonnent la réalisation d'une COS.

Nous notons par ailleurs, la forte présence d'une dépendance au contexte en situation de COS. D'après nos analyses, la perception de la réussite de l'exposé peut dépendre en partie de l'intérêt que porte l'auditoire au sujet présenté. Cette perception nous interpelle, car elle montre combien l'élément «auditoire», comme élément externe à soi, échappe au contrôle du présentateur, ou tout au moins devient un élément perturbateur de la réussite d'une COS. Cette dépendance au contexte a donc un impact réel sur le SEPss.

Rappelons que la dépendance au contexte en lien avec le SEP réfère dans cette étude à trois éléments: à l'intérêt intrinsèque de l'auditoire envers le sujet présenté, à la composition de l'auditoire (devant la classe complète, devant un petit groupe, devant des inconnus, etc.) et à la permission donnée ou non par l'enseignant d'utiliser du matériel de soutien (fiche de notes ou présentation PowerPoint). Pris dans un sens plus large, la dépendance au contexte pourrait également inclure d'autres éléments extérieurs au 


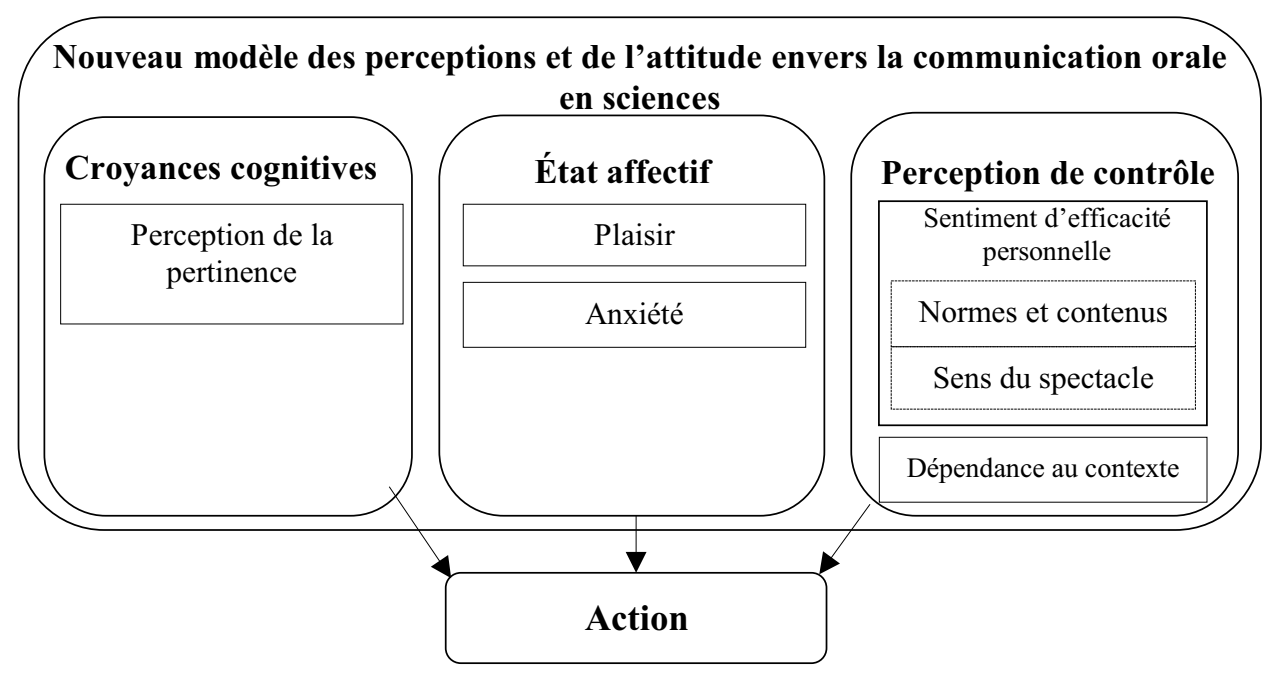

Fig. 3 Nouveau modèle théorique des Perceptions et de l'attitude envers la communication orale en sciences (NPACOS), adapté de van Aalderen-Smeets et al. (2012)

présentateur: le format de l'exposé, l'évaluation (sommative ou formative) ou le fait que la présentation doive être réalisée individuellement ou en équipe. Dans cette perspective, la présence d'une dépendance au contexte rejoint les travaux d'autres chercheurs (Marec et al., 2019; Van Aalderen-Smeets et al., 2012) qui la révèlent sous la forme de conditions externes au sujet à l'étude.

Ces considérations nous amènent à préciser la dimension de perception de contrôle de notre nouveau modèle désormais intitulé NPACOS en y intégrant la dépendance au contexte, comme représenté à la Fig. 3. La dépendance au contexte, qui faisait partie du modèle de l'attitude de van Aalderen-Smeets et ses collègues (2012) sous la dimension Perception de contrôle, est donc ajoutée à notre modèle, bien qu'aucun item du questionnaire PACOS n'ait été pensé pour l'évaluer spécifiquement. Il demeure que les observations menées en entretiens avec les étudiants nous autorisent à confirmer qu'il s'agit bel et bien d'un facteur important pour l'attitude envers la COS.

\section{Apports, limites et pistes de recherche}

Notre recherche a montré que le SEP s'exprime en deux aspects en COS: le SEP normes et contenu concerne la capacité à rendre un discours formellement correct, tant au niveau de la langue que du contenu scientifique, tandis que le SEP sens du spectacle est la croyance qu'on peut donner une présentation orale en sciences qui saura intéresser l'auditoire. Grâce à l'emploi d'une méthodologie mixte, les résultats issus des entretiens ont précisé les résultats du questionnaire PACOS en apportant une analyse éclairante sur la complexité du SEP en COS. Notre étude a démontré les éléments explicatifs du SEPnc et du SEPss et la relation qu'ils entretiennent. De plus, la «dépendance au contexte» comme facteur contribuant au SEP se joint à la dimension de la perception de contrôle dans le modèle théorique NPACOS, venant ainsi le rendre plus opératoire.

Comme limites à cette recherche, mentionnons que les données proviennent d'un échantillon composé d'étudiants du niveau collégial, niveau d'enseignement préuniversitaire québécois. Les chercheurs de l'étranger seront probablement intéressés à vérifier nos conclusions dans leur propre contexte éducatif. Il demeure que le champ de recherche sur l'oral en sciences nous permet de trouver des similarités entre les différents contextes et le nôtre, notamment au sujet d'un manque systématique de l'enseignement de la COS au postsecondaire (Chan, 2011) et de l'influence importante du SEP en communication orale 
(Demir, 2017). Une autre limite de notre étude concerne son échantillon dit de convenance, car constitué d'étudiants ayant accepté de répondre au questionnaire et d'accorder un entretien téléphonique. Les étudiants les moins à l'aise à l'oral ont peut-être refusé de participer aux entretiens téléphoniques. Pour mitiger cette limite, la stratification de l'échantillonnage des entretiens par grappes était essentielle et nous a permis de solliciter une variété somme toute représentative de participants pour les entretiens. Dans le même ordre d'idée, comme les étudiants qui ont répondu au questionnaire PACOS à la fin de leur parcours collégial sont ceux qui ont persévéré et réussi leur formation collégiale, il est probable qu'ils possédaient déjà au départ un SEPnc et un SEPss plus élevés. Ceci n'empêche pas que les résultats obtenus pour le troisième objectif sur l'évolution des deux aspects du SEP soient valables, mais ceux-ci sont limités aux étudiants qui complètent leur formation.

Comme piste de recherche, il semble intéressant d'étudier le lien entre la dépendance au contexte et d'autres facteurs du modèle NPACOS, dont l'anxiété et son impact sur la COS. En effet, on peut vérifier et comparer le niveau de stress en situation de COS selon des conditions variées, par exemple lors d'un exposé formel sommatif réalisé seul et sans support matériel ou lors d'un exposé formatif réalisé en équipe devant uniquement l'enseignant.

Il pourrait aussi être pertinent de regarder comment l'attitude des étudiants envers la COS se traduit dans une situation réelle de COS (dimension de l'action dans le modèle NPACOS), par exemple en regardant la maitrise de cette compétence et la qualité de la performance réalisée.

\section{Note sur l'éthique de la recherche}

Ce projet de recherche a reçu l'approbation des comités d'éthique à la recherche de tous les cégeps desquels provenaient les participants, avant le début du recrutement des participants. Toutes les procédures exécutées dans les études auxquelles ont participé des êtres humains ont été exécutées selon les normes approuvées par les comités de recherche éthique pertinents au niveau institutionnel ou national et conformément aux normes de la Déclaration d'Helsinki de 1964 (et amendements ultérieurs) ou à des normes comparables.

Funding Les auteurs tiennent à remercier le Programme d'aide à la recherche sur l'enseignement et l'apprentissage (PAREA) du Ministère de l'Éducation et de l'Enseignement supérieur du Québec pour le financement de la recherche décrite dans cet article (projet PA2017-016).

\section{Declarations}

Conflit D'intérêts Au nom de tous les auteurs, l'auteur correspondant déclare qu'il n'y a pas de conflit d'intérêts.

\section{Références}

Ajzen, I., \& Fishbein, M. (2005). The Influence of Attitudes on Behavior. Dans The handbook of attitudes (Vol. 173, p. 173-221).

Alsop, S., \& Bencze, L. (2014). Activism ! Toward a more radical science and technology education. Dans Activist Science and Technology Education (p. 1-19). Springer. https://doi.org/10.1007/978-94-007-4360-1

Applebee, M. S., Johanson, A. P., Lawler-Sagarin, K. A., Losey, E. N., \& Munro-Leighton, C. (2018). The Three-Minute Slide as an Effective Tool for Developing Oral Communication Skills. Journal of Chemical Education, 95(8), 1419-1422.

Bandura, A. (2007). Auto-efficacité : Le sentiment d'efficacité personnelle (J. Lecomte, Trad.; 2e éd.). De Boeck.

Bandura, A. (2009). La théorie sociale cognitive : Une perspective agentique. Dans P. Carré \& F. Fenouillet (Éds.), Traité de psychologie de la motivation (p. 15-45). Dunod. 
Bjorklund, S. A., \& Colbeck, C. L. (2001). The View from the Top : Leaders' Perspectives on a Decade of Change in Engineering Education. Journal of Engineering Education, 90(1), 13-19. https://doi.org/10.1002/j.2168-9830.2001. tb00562.x

Blair, E., \& Blair, J. (2014). Applied Survey Sampling. SAGE Publications Inc.

Blanchet, P.-A. (2016). Les choix de contenus d'enseignement et d'évaluation de la compétence orale dans les plans de cours de français du collégial : Portraits de trois cégeps [Thèse de doctorat, Université de Sherbrooke]. https://savoirs.usherbrooke. ca/handle/11143/8730

Blanchet, P.-A. (2017). Un aperçu des pratiques d'enseignement de l'oral au collégial. Correspondance, 23(4). https:// correspo.ccdmd.qc.ca/index.php/document/un-apercu-des-pratiques-denseignement-de-loral-au-collegial/

Boutin, G. (2018). L'entretien de recherche qualitatif (2e éd.). PUQ. https://www.puq.ca/catalogue/livres/entretien-recherchequalitatif-edition-3258.html

Boyer, P. (2012). La compétence morphographique d'élèves de première secondaire : L'effet du biais d'évaluation de son efficacité personnelle sur la performance [Université du Québec à Montréal]. https://archipel.uqam.ca/5352/

Boyer, P., Messier, G., Dumais, C., \& Viola, S. (2018). Le profil motivationnel d'étudiants en formation initiale à l'enseignement au Québec au regard du développement de la compétence à communiquer oralement : Premiers résultats d'une étude longitudinale. Revue internationale de pédagogie de l'enseignement supérieur, 34(3). https://doi.org/10.4000/ripes.1717

Brownell, S. E., Price, J. V., \& Steinman, L. (2013). Science Communication to the General Public : Why We Need to Teach Undergraduate and Graduate Students this Skill as Part of Their Formal Scientific Training. Journal of Undergraduate Neuroscience Education, 12(1), E6-E10.

Chan, V. (2011). Teaching oral communication in undergraduate science : Are we doing enough and doing it right? Journal of Learning Design, 4(3), 71-79.

Cleveland, L. M., \& Reinsvold, R. J. (2017). Development of Oral Communication Skills by Undergraduates that Convey Evolutionary Concepts to the Public. Journal of Microbiology \& Biology Education, 18(1). https://doi. org/10.1128/jmbe.v18i1.1227

Cohen, J. (1988). Statistical power analysis for the behavioral sciences. Lawrence Erlbaum Associates.

Creswell, J. W., \& Creswell, J. D. (2018). Research Design : Qualitative, Quantitative, and Mixed Methods Approaches (5e éd.). SAGE Publications, Inc. https://us.sagepub.com/en-us/nam/research-design/book255675

Demir, S. (2017). An Evaluation of Oral Language : The Relationship between Listening, Speaking and Self-efficacy. Universal Journal of Educational Research, 5(9), 1457-1467. https://doi.org/10.13189/ujer.2017.050903

Desombre, C., Durand-Delvigne, A., Heutte, J., \& Brasselet, C. (2016). Sentiment d'efficacité personnelle des garçons et des filles : L'importance des conditions de travail. Enfance, $N^{\circ} 3(3), 287-298$

Dionne, L. (2018). L'analyse qualitative des données. Dans T. Karsenti \& L. Savoie-Zajc (Éds.), La recherche en éducation. Étapes et approches (4e éd., p. 317-342). Les Presses de l'Université de Montréal.

Dumais, C. (2017). Communiquer oralement : Une compétence à développer au collégial. Pédagogie collégiale, 31(1), 13-19.

Dumais, C., \& Ostiguy, L. (2019). Développer la compétence à communiquer oralement au collégial : Les caractéristiques de la langue parlée. Correspondance, 25(1). https://correspo.ccdmd.qc.ca/index.php/document/developperla-competence-a-communiquer-oralement-au-collegial-les-caracteristiques-de-la-langue-parlee/

Dumont, M., LeClerc, D., \& Deslandes, R. (2003). Ressources personnelles et détresse psychologique en lien avec le rendement scolaire et le stress chez des élèves de quatrième secondaire. Canadian Journal of Behavioural Science / Revue canadienne des sciences du comportement, 35(4), 254-267. https://doi.org/10.1037/h0087206

Dwyer, K. K., \& Fus, D. A. (2002). Perceptions Of Communication Competence, Self-Efficacy, And Trait Communication Apprehension : Is There an Impact on Basic Course Success? Communication Research Reports, 19(1), 29-37. https://doi.org/10.1080/08824090209384829

Edmondston, J. E., Dawson, V., \& Schibeci, R. (2010). Undergraduate Biotechnology Students' Views of Science Communication. International Journal of Science Education, 32, 2451-2474. https://doi.org/10.1080/09500690903514598

Field, A. (2017). Discovering Statistics Using SPSS : North American Edition (5e éd.). SAGE Publications, Inc.

Fortin, M.-F., \& Gagnon, J. (2015). Fondements et étapes du processus de recherche: Méthodes quantitatives et qualitatives (3e éd.). Chenelière Éducation.

Gaudreau, N., Royer, É., Beaumont, C., \& Frenette, É. (2012). Le sentiment d'efficacité personnelle des enseignants et leurs pratiques de gestion de la classe et des comportements difficiles des élèves. Canadian Journal of Education / Revue canadienne de l'éducation, 35(1), 82-101.

Huerta, M., Goodson, P., Beigi, M., \& Chlup, D. (2017). Graduate students as academic writers : Writing anxiety, self-efficacy and emotional intelligence. Higher Education Research \& Development, 36(4), 716-729. https:// doi.org/10.1080/07294360.2016.1238881

Jones, M. G., \& Leagon, M. (2014). Science teacher attitudes and beliefs. Dans N. Lederman \& S. Abell (Éds.), Handbook of research on science education (Vol. 2, p. 830-843). Routeledge.

Karsenti, T., \& Savoie-Zajc, L. (2018). La recherche en éducation : Étapes et approches (4e éd.). Les Presses de l’Université de Montréal. 
Khatib, F. M. M., \& Maarof, N. (2015). Self-efficacy Perception of Oral Communication Ability among English as a Second Language (ESL) Technical Students. Procedia - Social and Behavioral Sciences, 204, 98-104. https:// doi.org/10.1016/j.sbspro.2015.08.121

Koch, P., \& Oesterreicher, W. (2001). Langage oral et langage écrit. Dans Lexikon der romanistischen Linguistik. Band I, 2 : Methodologie (p. 584-627). Max Niemeyer Verlag.

Kulgemeyer, C. (2018). Impact of Secondary Students' Content Knowledge on Their Communication Skills in Science. International Journal of Science and Mathematics Education, 16(1), 89-108. https://doi.org/10.1007/ s10763-016-9762-6

Kulgemeyer, C., \& Schecker, H. (2013). Students explaining science-Assessment of science communication competence. Research in Science Education, 43(6), 2235-2256.

Langlois, S., \& Cormier, C. (soumis). Sentiment d'efficacité personnelle à l'oral d'étudiants postsecondaires en sciences : Élaboration de l'échelle Perception et attitude envers la communication orale scientifique (PACOS). Mesure et évaluation en éducation.

Marec, C.-É., Tessier, C., \& Langlois, S. (2019). Évolution du sentiment d'efficacité des enseignants du primaire ayant suivi un programme de formation et d'accompagnement en sciences et technologie. Canadian Journal of Science, Mathematics and Technology Education, 19. https://doi.org/10.1007/s42330-019-00058-6

Masson, J., \& Fenouillet, F. (2013). Relation entre sentiment d'efficacité personnelle et résultats scolaires à l'école primaire : Construction et validation d'une échelle. https://doi.org/10.4074/S0013754513004047

McLaren, I. (2019). Science Students' Responses to an Oral Communication Skills Development Initiative : Attitude and Motivation. International Journal of Teaching and Learning in Higher Education, 31(1), 73-85.

MEES. (2017). Composantes de la formation générale - Extraits des programmes d'études conduisant au diplôme d'études collégiales (DEC). Gouvernement du Québec.

MEES. (2021). Effectif à l'enseignement collégial selon diverses variables, au trimestre d'automne. Environnement informationnel, système Socrate. http://www.bdso.gouv.qc.ca/

Mercer, S., \& Williams, M. (2014). Multiple Perspectives on the Self in SLA. Multilingual Matters.

Mercer-Mapstone, L. D., \& Kuchel, L. (2015). Teaching Scientists to Communicate : Evidence-based assessment for undergraduate science education. International Journal of Science Education, 37(10), 1613-1638. https://doi.org/ 10.1080/09500693.2015.1045959

Mercer-Mapstone, L. D., \& Matthews, K. E. (2017). Student Perceptions of Communication Skills in Undergraduate Science at an Australian Research-Intensive University. Assessment \& Evaluation in Higher Education, 42(1), 98-114. https://doi.org/10.1080/02602938.2015.1084492

Olsen, R. V., \& Lie, S. (2011). Profiles of Students' Interest in Science Issues around the World : Analysis of data from PISA 2006. International Journal of Science Education, 33(1), 97-120. https://doi.org/10.1080/09500693.2010. 518638

Osborne, J., Simon, S., \& Collins, S. (2003). Attitudes towards science : A review of the literature and its implications. International Journal of Science Education, 25(9), 1049-1079. https://doi.org/10.1080/0950069032000032199

Papin, K. (2019). La contribution des tâches de réalité virtuelle au désir de communiquer en français langue seconde à l'extérieur de la salle de classe à Montréal [Thèse de doctorat]. Université de Montréal.

Skaalvik, E. M., \& Skaalvik, S. (2004). Self-concept and self-efficacy : A test of the internal/external frame of reference model and predictions of subsequent motivation and achievement. Psychological Reports, 95(3 Pt 2), 1187-1202. https://doi.org/10.2466/pr0.95.3f.1187-1202

Smith, E. V. Jr., Wakely, M. B., De Kruif, R. E. L., \& Swartz, C. W. (2003). Optimizing rating scales for self-efficacy (and other) research. Educational and Psychological Measurement, 63(3), 369-391. https://doi.org/10.1177/ 0013164403063003002

Usher, E. L., \& Pajares, F. (2006). Sources of academic and self-regulatory efficacy beliefs of entering middle school students. Contemporary Educational Psychology, 31(2), 125-141. https://doi.org/10.1016/j.cedpsych.2005.03.002

Van Aalderen-Smeets, S. I., Walma van der Molen, J. H., \& Asma, L. J. F. (2012). Primary teachers' attitudes toward science : A new theoretical framework. Science Education, 96(1), 158-182. https://doi.org/10.1002/sce.20467

Van Aalderen-Smeets, S., \& Walma van der Molen, J. H. (2013). Measuring Primary Teachers' Attitudes Toward Teaching Science : Development of the Dimensions of Attitude Toward Science (DAS) Instrument. International Journal of Science Education, 35(4), 577-600. https://doi.org/10.1080/09500693.2012.755576

Vrugt, A., Oort, F. J., \& Waardenburg, L. (2009). Motivation of men and women in mathematics and language. International Journal of Psychology, 44(5), 351-359. https://doi.org/10.1080/00207590802324831

Publisher's Note Springer Nature remains neutral with regard to jurisdictional claims in published maps and institutional affiliations. 